\title{
Diferencias fisicoquímicas de suelo suplementado con pilas eléctricas ${ }^{1}$
}

\section{Physical and chemical differences of the supplemented soil with electric batteries}

\author{
N. C. Bonilla, M. J. Rojas-Bahamón, D. F. Arbeláez \\ Recibido: febrero 22 de 2019 - Aceptado: septiembre 30 de 2019.
}

\begin{abstract}
Resumen - Existen prácticas de campesinos en el departamento del Caquetá y testimonios de personas en la ciudad de Florencia que involucran el uso de pilas eléctricas como base del abono orgánico para plantas. Frente a la hipótesis que las pilas eléctricas pueden contribuir al desarrollo saludable de una planta, se planteó como objetivo del presente estudio, evaluar la incidencia de las pilas eléctricas en las propiedades físicas y químicas de una muestra de suelo destinado a la siembra de fríjol. El experimento consistió en un diseño completamente al azar (DCA) con arreglo factorial $1 \times 4$, donde el factor A corresponde fríjol y el factor $B$ a la variedad de pilas eléctricas (genéricas y alcalinas) con tres repeticiones. En cada tratamiento se determinó: $\mathrm{pH}$, acidez intercambiable (A.I.), carbono orgánico (C.O), cenizas, color y conductividad. El estudio permitió evidenciar que la adición de pilas eléctricas al suelo favorece la absorción de la energía solar, haciendo que la temperatura media del suelo tienda a ser más elevada lo que tendería a promover una germinación rápida en las semillas, aspecto positivo en la etapa germinativa de estas. No obstante, puede incidir en la baja liberación de nitrógeno al suelo, en sus fases posteriores al crecimiento.
\end{abstract}

Palabras clave - pilas eléctricas, materia orgánica, sustrato, nitrógeno.

Abstract-There are peasant practices in the department of Caquetá and testimonies of people in the city of Florence that involve the use of electric batteries as the basis of organic fertilizer for plants.

${ }^{1}$ Producto derivado del proyecto de investigación "diferencias fisicas y químicas que existen en el suelo enriquecido con pilas eléctricas y otro con ausencia".de la Institución Educativa Jorge Eliécer Gaitán.

N. C. Bonilla, Universidad de la Amazonía, Florencia, Caquetá, Colombia, email: n.bonilla@udla.edu.co

M. J. Rojas-Bahamón, Institución Educativa Jorge Eliécer Gaitán, Florencia, Caquetá, Colombia, email: mjulissa@gmail.com.

D. F. Arbeláez, Universidad de la Amazonia, Florencia, Caquetá, Colombia, email: dfaca@hotmail.com.

Como citar este artículo: Bonilla, N. C., Rojas-Bahamón, M. J. Arbeláez, D. F. Diferencias fisicoquímicas de suelo suplementado con pilas eléctricas, Entre Ciencia e Ingeniería, vol. 13, no. 26, pp.68-73, julio-diciembre 2019. DOI: https://doi.org/10.31908/19098367.1164.

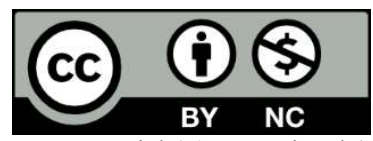

Attribution-NonCommercial 4.0 Intenational (CC By-NC 4.0)
Faced with the hypothesis that electric batteries can contribute to the healthy development of a plant, the objective of the present study was to evaluate the incidence of electric batteries in the physical and chemical properties of a soil sample destined to plant beans. The experiment consisted of a completely randomized design (DCA) with a $1 \times 4$ factorial arrangement, where factor $A$ corresponds to beans, and factor $B$ corresponds to the variety of electric batteries (generic and alkaline) with three repetitions. In each treatment, it was determined: $\mathrm{pH}$, exchangeable acidity (A.I.), organic carbon (C.O), ashes, color, and conductivity. The study showed that the addition of electric batteries to the soil favors the absorption of solar energy, making the average soil temperature tend to be higher, which would tend to promote rapid germination in the seeds, positive aspect in the germination stage of this. However, it can affect the low release of nitrogen to the soil, in its post-growth phases.

Keywords - electric batteries, organic matter, substrate, nitrogen.

\section{INTRODUCCIÓN}

T A contaminación por residuos tecnológicos es una

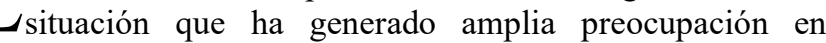
diferentes gobiernos a nivel mundial [1]. En Colombia particularmente, la literatura y normatividad respecto al tema es incipiente, pues sólo hasta agosto de 2010 el Estado Colombiano emitió la política para establecer los sistemas de recolección selectiva y gestión ambiental de residuos de computadoras y/o periféricos (Resolución 1512 de 2010) y la de residuos de pilas y/o acumuladores de acuerdo con la Resolución 1297 de 2010. Varios exponentes del tema expresan que la disposición y el tratamiento de residuos tecnológicos debe hacerse directamente con empresas especializadas en el asunto [2] [3] [4] [5]. Sin embargo, se evidencia en el proceder habitual de las personas, que la disposición final de artefactos y elementos tecnológicos se ha convertido en un asunto doméstico.

En esa vía, elementos tan comunes y cotidianos como las pilas eléctricas son depositadas en la basura domiciliaria luego de ser usadas. Así mismo, en relación con las pilas, algunos estudios han arrojado resultados que indican que estos dispositivos son fabricados con elementos químicos considerados como tóxicos y que su disposición final en basura 
doméstica o a cielo abierto podría causar graves problemas al medio ambiente y a la salud del ser humano. [6], sin embargo, la Asociación Nacional de Industriales [7] refiere estudios de la Universidad de Fukuoka Japón, la Universidad de Waterloo, Ontario, Canadá y la Universidad de Liege, Bélgica, que afirman que el desecho de pilas (alcalinas y de zinc-carbón) no representan un riesgo para la población o recursos naturales, así mismo no objetan el desecho de pilas sin mercurio, ni cadmio, ni plomo en rellenos sanitarios [9][10][11][12].

A pesar de la postura dual que hay entre los diferentes estudios frente al impacto ambiental, lo único que queda claro en primer lugar, es que las pilas deben tener una disposición segura a través de entidades competentes en el tema, con puntos de recolección y centros de acopio específicos. Y, en segundo lugar, que existe en la población un desconocimiento generalizado respecto al tema y al desecho de esos materiales.

En Colombia, el panorama respecto al tratamiento de las pilas eléctricas implica el proceso de recolección en centros de acopio, para lo cual existen empresas encargadas de recolectar pilas y darles disposición final segura. A nivel nacional se presentan puntos de recolección en las principales ciudades del país, sin embargo, en la ciudad de Florencia solo existen dos puntos y ninguno en los municipios del Caquetá.

En consideración de lo anterior, los hábitos en este sector del país, sugieren que los ciudadanos desechan las pilas en la basura, a cielo abierto o las entierran, puesto que algunas inspecciones oculares en algunos sectores del río Hacha, permitieron encontrar pilas en las orillas del río, en los restos de basura e incluso dentro del agua. De igual manera, respecto a la disposición de las pilas, indagaciones realizadas a algunos campesinos de zonas rurales de San Vicente del Caguán permitieron descubrir prácticas que manifiestan que las pilas "ayudan" a mejorar la apariencia de plantas como la sábila y el arroz, mejorando particularmente en este último, la apariencia de tallos, hojas y evitando que la planta se marchite. Así mismo, testimonios de personas en la ciudad de Florencia, coincidieron en el manejo de pilas como "fertilizante", que produce, en corto plazo, un efecto que los entrevistados denotaron como especial sobre las plantas, dado que mejoran la apariencia física de la misma volviendo sus hojas más verdes.

Por eso, en consideración de las particularidades del contexto a estudiar, la poca literatura existente al respecto, las experiencias y las conjeturas manifestadas en algunos pobladores de sectores rurales del Caquetá frente a la hipótesis de que las pilas eléctricas pueden constituirse en un elemento importante que puede contribuir al desarrollo saludable de un organismo, se planteó como objetivo del presente estudio, evaluar la incidencia de las pilas eléctricas en las propiedades físicas y químicas de una muestra de suelo destinado a la siembra de fríjol.

\section{MATERIALES Y MÉTODOS}

\section{A. Ubicación geográfica}

El estudio se realizó en el vivero de la Sede San Juan Bosco de la Institución Antonio Ricaurte del municipio de Florencia Caquetá, Colombia con coordenadas geográficas $1^{\circ} 37^{\prime} 10,38^{\prime \prime} \mathrm{N}$ y $75^{\circ} 36^{\prime} 23,07^{\prime \prime} \mathrm{W}$ a $242 \mathrm{msnm}$, con una humedad relativa del $86 \%$, una temperatura promedio de $26^{\circ} \mathrm{C}$, precipitación promedio anual de $3.695 \mathrm{~mm}$ y un brillo solar de 4,6 horas/día.
Las pruebas de laboratorio se realizaron en el laboratorio de Geociencias del Centro de Investigación Macagual "Cesar Augusto Estrada" de la Universidad de la Amazonia.

\section{B. Ruta metodológica}

El experimento consistió en un diseño completamente al azar (DCA) con arreglo factorial 1 x 4, donde el factor A correspondió a semillas de fríjol y el factor B a la variedad de pilas eléctricas, con tres repeticiones.

\section{Factor A: Híbrido}

H1: Fríjol (Phaseolus vulgaris)

\section{Factor B: Variedad de pilas}

M1: Blanco, sustrato sin pilas.

M2: sustrato con pilas genéricas.

M3: sustrato con pilas alcalinas.

M4: sustrato con pilas genéricas y alcalinas.

\section{- Unidad experimental}

La Unidad Experimental (UE) correspondió a materas con capacidad de $25 \mathrm{~kg}$ donde se distribuyó un sustrato artificial y 10 semillas de fríjol (Phaseolus vulgaris) con sus respectivas repeticiones y variedades de pilas.

\section{- Preparación de las muestras}

Se colectaron las muestras de suelo en sendos vasos de precipitado de $250 \mathrm{~mL}$. Se pesó el suelo húmedo, posteriormente se colocó cada una de las muestras en el horno a una temperatura de $105{ }^{\circ} \mathrm{C}$ por 24 horas. Una vez secas las muestras, se pasaron por un tamiz de malla $2 \mathrm{~mm}$ con el objeto de obtener un tamaño homogéneo de partícula.

\section{C.Determinación de parámetros fisicoquímicos.}

La determinación de los parámetros fisicoquímicos se realizó en el laboratorio de Geociencias del Centro de Investigación Macagual "Cesar Augusto Estrada" de la Universidad de la Amazonia, siguiendo los protocolos que se describen a continuación: el pH del suelo se midió en una solución suelo: agua, $1: 1(\mathrm{p} / \mathrm{v})$ [13] , la acidez intercambiable (A.I) se obtuvo por titulación con $\mathrm{NaOH} 0,1 \mathrm{~N}$ [14], el carbono orgánico (C.O) se estimó con el método de Walkley \& Black [15] [16], las cenizas se determinaron mediante el método de calcinación, el color se determinó mediante el uso de la tabla Munsell y la conductividad eléctrica se determinó en términos de sales solubles [17].

Determinación de $p H$. Para la determinación del $\mathrm{pH}$ lo primero que se hizo fue calibrar el peachímetro. Se pesó $10 \mathrm{~g}$ de suelo seco y pasado por tamiz de malla $2 \mathrm{~mm}$ en beaker plástico, se agregó $10 \mathrm{ml}$ de agua destilada y desionizada, se homogenizó con el agitador mecánico durante $10 \mathrm{~min}$ en intervalos de $15 \mathrm{~min}$ durante una hora. Cumplido este último tiempo se agitó vigorosamente la muestra por 30 segundos e inmediatamente se tomó el valor de $\mathrm{pH}$ de la suspensión formada. Se lavó y enjuagó el electrodo al terminar cada lectura. Se repitió el procedimiento para cada una de las muestras.

Determinación de acidez intercambiable (A.I.). Se pesó $10 \mathrm{~g}$ de suelo seco y tamizado, en balanza de triple brazo, se agregó 
al tubo de centrifuga, se adicionó $80 \mathrm{ml}$ de $\mathrm{KCl} 1 \mathrm{~N}$ y agitó continuamente por $5 \mathrm{~min}$. El sobrenadante se pasó a erlenmeyer de $250 \mathrm{ml}$. El residuo sólido fue eliminado. Al sobrenadante se adicionaron dos gotas de fenolftaleína al $0.1 \%$ y se tituló con $\mathrm{NaOH} 0.1 \mathrm{~N}$ hasta aparición de tonalidad rosada pálida. Se anotó el volumen $(\mathrm{mL})$. El blanco estuvo conformado por 80 $\mathrm{ml}$ de $\mathrm{KCl} 1 \mathrm{~N}$ más 2 gotas de fenolftaleína $0.1 \%$.

Fórmula para determinar acidez intercambiable

$$
\text { A. } I=\frac{\text { Volumen } \mathrm{NaOH}(\mathrm{ml}) \times \text { Nomalidad }\left(\mathrm{meq} \mathrm{ml}^{-1}\right) \mathrm{del} \mathrm{NaOH}}{\text { Masa de la muestra }(\mathrm{g})} \times 100
$$

Determinación de carbono orgánico (C.O). Se conectó la plancha de calentamiento. Se pesó $0,1 \mathrm{~g}$ de suelo seco y de tamaño de partícula $2 \mathrm{~mm}$. Se agregó la muestra de suelo en un erlenmeyer de $250 \mathrm{~mL}$. Se adicionaron $10 \mathrm{ml}$ de $\mathrm{K}_{2} \mathrm{Cr}_{2} \mathrm{O}_{7} 1 \mathrm{~N}$ con probeta y se agitó para dispersar el suelo. Se colocó el erlenmeyer a chorro de agua, se adicionó lentamente $10 \mathrm{ml}$ de $\mathrm{H}_{2} \mathrm{SO}_{4}$ concentrado y se agitó suavemente (la reacción es fuertemente exotérmica).

Posteriormente, se colocó el erlenmeyer en la plancha caliente por 15 min sin agitar; luego, se retiró el erlenmeyer de la plancha y se dejó enfriar. Luego, se colocó el erlenmeyer a chorro de agua y se adicionaron lentamente $100 \mathrm{ml} \mathrm{de} \mathrm{H}_{2} \mathrm{O}$ destilada y desionizada (la disolución es exotérmica). Luego de esto, se dejó enfriar la muestra para agregar 5 gotas de ferroína (indicador). Luego se tituló la muestra con $\mathrm{FeSO}_{4} 1 \mathrm{~N}$, el cambio de color verde a marrón indica la terminación de la titulación.

Finalmente, se anotó el volumen $(\mathrm{mL})$, se repitió el mismo procedimiento para cada muestra. El blanco estuvo conformado por: $10 \mathrm{ml}$ de dicromato de potasio, $\mathrm{K}_{2} \mathrm{Cr}_{2} \mathrm{O}_{7}$ más $10 \mathrm{ml} \mathrm{H}_{2} \mathrm{SO}_{4}$.

Fórmula para determinar carbono orgánico (C.O)

$$
\begin{gathered}
C . O=\frac{\left(V_{1} N_{1}-V_{2} N_{2}\right)}{W} \times 0,003 \times 1,724 \times 1,32 \times 100 \\
C . O=\frac{\left(V_{1} N_{1}-V_{2} N_{2}\right)}{W} \times 0,68
\end{gathered}
$$

Donde,

$\mathrm{V}_{1}$ : $\mathrm{ml}$ de $\mathrm{K}_{2} \mathrm{Cr}_{2} \mathrm{O}_{7}$

$\mathrm{V}_{2}: \mathrm{ml}$ de $\mathrm{FeSO}_{4} .7 \mathrm{H}_{2} \mathrm{O}$ gastados

$\mathrm{W}$ : peso de la muestra

$\mathrm{N}_{1}$ : Normalidad de $\mathrm{K}_{2} \mathrm{Cr}_{2} \mathrm{O}_{7}$

$\mathrm{N}_{2}$ : Normalidad del $\mathrm{FeSO}_{4} \cdot 7 \mathrm{H}_{2} \mathrm{O}$

$$
\frac{C . O}{\text { N.total }}=\text { nivel de mineralización media }
$$

Determinación de cenizas. Para la determinación de cenizas se calcinó el crisol a $550{ }^{\circ} \mathrm{C}$ por 2 horas, se dejó enfriar y se pesó $\left(\mathrm{P}_{1}\right)$, inmediatamente se agregó cerca de $1.5 \mathrm{~g}$ de suelo seco y tamizado a $2 \mathrm{~mm}$, se pesó sin tarar $\left(\mathrm{P}_{2}\right)$. Se colocó en la mufla a $550^{\circ} \mathrm{C}$ por $2 \mathrm{~h}$, se sacó el crisol de la mufla, se dejó enfriar y nuevamente se pesó $\left(\mathrm{P}_{3}\right)$.

Fórmula para determinar cenizas (Contenido de minerales)

$$
\text { \%ceniza }=\frac{P_{3}-P_{1}}{P_{2}} \times 100
$$

Determinación del color en seco.Se sacó, con ayuda de una espátula limpia, alrededor de $1 \mathrm{~g}$ de la muestra seca, cuarteada, rotulada y almacenada. Se procedió a usar la tabla de Munsell para determinar, por triplicado, nombre, hue, value y chroma del color.

Determinación de conductividad. Se utilizó una muestra de $100 \mathrm{~g}$ suelo seco y tamizado en beaker. Se colocaron $100 \mathrm{ml}$ de agua en la probeta y agregaron pequeñas cantidades de agua destilada al suelo hasta formar una pasta brillante y fluida. Se anotó el volumen gastado. Luego se agregó un volumen de agua igual al previamente anotado. Se dejó en reposo durante 2 horas. Se centrifugó y separó el sobrenadante y se procedió a medir la conductividad. El proceso se repitió para cada muestra. Fórmula para convertir conductividad (C) de $\mu \mathrm{S} / \mathrm{cm}$ a dS/m.

$$
\frac{245.6 \mu \mathrm{S} / \mathrm{cm}}{1000}=0,2456 \mathrm{dS} / \mathrm{m}
$$

Fórmula para determinar la salinidad del suelo

Salinidad $=\frac{5 C}{3}$ gramos de sales desuelo disuelto por litro de agua

Determinación de iones en extracto acuoso. La determinación de algunos iones (cloruros, nitratos, sulfatos, carbonatos, sulfuros, calcio, sodio, potasio) en extracto acuoso, fueron determinados mediante un tamizaje químico de acuerdo con la metodología de Álvarez R. y otros [18].

\section{RESULTADOS Y DISCUSIÓN}

La interpretación de los resultados se realizó atendiendo a las consideraciones generales propuestas por el Instituto Geográfico Agustín Codazzi, subdirección de agrología.

Teniendo en cuenta los datos de la Tabla I, se pudo observar que el $\mathrm{pH}$ para todos los tratamientos de suelo estuvo en el rango medianamente ácido, excepto el que no contenía pilas, cuyo valor de $\mathrm{pH}$ fue mayor. Esto significa que las pilas aportan al suelo iones que hacen que el $\mathrm{pH}$ baje, aunque no de una manera significativa.

En términos generales, dependiendo del $\mathrm{pH}$ del suelo se puede: facilitar la substitución del calcio y del magnesio; afectar la solubilidad del hierro, aluminio, manganeso y otros elementos menores; afectar la asimilabilidad del fósforo y la actividad de los microorganismos.

TABLA I

VALOR DE PH PARA LOS DISTINTOS TRATAMIENTOS.

\begin{tabular}{cccc}
\hline \multicolumn{4}{c}{$\mathbf{p H}$} \\
\hline $\mathbf{M}_{\mathbf{1}}$ & $\mathbf{M}_{\mathbf{2}}$ & $\mathbf{M}_{3}$ & $\mathbf{M}_{\mathbf{4}}$ \\
\hline 6,05 & 5,77 & 5,79 & 5,78 \\
\hline
\end{tabular}

Teniendo en cuenta que, la Capacidad de Intercambio de Cationes Efectiva (CICE) o acidez intercambiable representa la sumatoria de $\mathrm{Ca}+\mathrm{Mg}+\mathrm{K}+\mathrm{Al}+\mathrm{H}$ en el suelo y que valores de saturación de acidez mayores al $10 \%$ pueden afectar negativamente el crecimiento de especies vegetales poco tolerantes a la presencia de $\mathrm{Al}$, mientras que el valor de $60 \%$ se 
considera como el máximo para especies tolerantes a la acidez del suelo, el valor deseable para la mayoría de las plantas oscila entre 10 y $25 \%$.

Se puede afirmar que la adición de pilas al suelo no afecta significativamente la capacidad de intercambio de cationes efectiva en el suelo, comparada con el blanco, pues, la acidez intercambiable está por debajo del $10 \%$, según los resultados de la Tabla II.

TABLA II

VALOR DE ACIDEZ INTERCAMBIABLE (A.I.) PARA LOS DISTINTOS TRATAMIENTOS.

(A.I) (meq- $g \times 100 \mathrm{~g}$ de muestra)

\begin{tabular}{ccccc}
\hline Blanco & $\boldsymbol{M}_{1}$ & $\boldsymbol{M}_{2}$ & $\boldsymbol{M}_{3}$ & $\boldsymbol{M}_{4}$ \\
\hline- & 0,175 & 1,4 & 1,575 & 1,575 \\
\hline
\end{tabular}

El carbono orgánico en el suelo hace que aumente la capacidad retentiva de agua al actuar como esponja. Además, la materia orgánica reduce las pérdidas de agua por escurrimiento y al mismo tiempo la erosión favoreciendo la infiltración. La presencia de materia orgánica en el suelo hace que el color oscuro prevalezca, facilitando la absorción de la energía solar, por lo que la temperatura media del suelo suele ser algo más elevada que aquellos suelos que presenta carencia de esta, lo que tiende a promover una germinación más rápida en las semillas (Fuentes, 1967). Aspecto positivo en la etapa germinativa de las semillas sembradas en un tipo de suelo con estas características.

La materia orgánica en el suelo regula el $\mathrm{pH}$, esto explica que el $\mathrm{pH}$ del suelo no hubiese cambiado sustancialmente en los tratamientos estudiados comparados con el blanco. Además, según Bastones (2004), la materia orgánica inmoviliza los metales pesados. Influye en la capacidad de intercambio catiónico (CIC), aumentándola y, por lo tanto, aumentando la fertilidad química del suelo. No obstante, puede incidir en la baja liberación de nitrógeno al suelo, aspecto que puede subsanarse con fertilizantes orgánicos ricos en nitrógeno.

$\mathrm{Al}$ analizar la relación $\mathrm{C} / \mathrm{N}$ en cada uno de los tratamientos de la presente investigación, se puede afirmar que tanto para el blanco como para los tratamientos $\mathrm{M}_{1} \mathrm{y}_{3}$, es alta y para el tratamiento $\mathrm{M}_{2}$, es muy alta como se puede notar en la Tabla III. La adición de pilas a cada uno de los tratamientos modifica sustancialmente el contenido de carbono orgánico en el suelo, siendo las pilas alcalinas el que más lo modifica. Cabe aclarar que, de acuerdo con las características visuales de los tratamientos, el carbono que aportan las pilas no fue totalmente asimilado, pues al tacto manchan la piel situación que no ocurre cuando se tiene un suelo rico en humus o materia orgánica.

Se puede observar que todas las muestras de suelo presentaron un alto porcentaje de materia orgánica. No obstante, el suelo suplementado con pilas alcalinas $\left(\mathrm{M}_{3}\right)$ presentó un mayor porcentaje con respecto a las otras muestras.

De acuerdo con los datos de cenizas para cada uno de los tratamientos en esta investigación se puede inferir que la adición de pilas genéricas al suelo no modifica substancialmente el contenido de ceniza o contenidos de minerales en el suelo, es decir, que el aporte en cuanto a minerales al suelo es insignificante (tabla IV).
TABLA III

PORCENTAJE PROMEDIO DE: CARBONO ORGÁNICO (C.O), MATERIA ORGÁNICA (M.O), NITRÓGENO TOTAL (N TOTAL), NITRÓGENO DISPONIBLE EN SUELO (N DISPONIBLE) Y RELACIÓN DE C.O/N TOTAL PARA LOS DISTINTOS TRATAMIENTOS.

\begin{tabular}{|c|c|c|c|c|c|}
\hline & Blanco & $\mathrm{M}_{1}$ & $\mathrm{M}_{2}$ & $\mathrm{M}_{3}$ & $\mathrm{M}_{4}$ \\
\hline$\%$ C.O & - & 13,04 & 13,04 & 17,72 & 12,51 \\
\hline$\% \mathrm{M} . \mathrm{O}$ & - & 22,48 & 22,48 & 30,55 & 21,56 \\
\hline$\% \mathrm{~N}$ total & - & 1,124 & 1,124 & 1,528 & 1,078 \\
\hline $\begin{array}{lr}\% & \mathrm{~N} \\
\text { disponible } & \text { en } \\
\text { suelo } & \\
\end{array}$ & - & 0,030 & 0,030 & $\begin{array}{c}0,041 \\
2\end{array}$ & 0,029 \\
\hline $\begin{array}{l}\text { Relación } \\
\text { C.O./N. total }\end{array}$ & - & 11,60 & 11,60 & 15,76 & 11,13 \\
\hline
\end{tabular}

TABLA IV

CENIZA (CONTENIDO DE MINERALES) PARA LOS DISTINTOS TRATAMIENTOS

\begin{tabular}{cccc}
\hline \multicolumn{5}{c}{$\%$ de ceniza } \\
\hline $\mathrm{M}_{1}$ & $\mathrm{M}_{2}$ & $\mathrm{M}_{3}$ & $\mathrm{M}_{4}$ \\
\hline 0,591 & 0,513 & 0,58 & 0,56 \\
\hline
\end{tabular}

El color de los suelos depende fundamentalmente de dos constituyentes: la materia orgánica y la materia mineral. La materia orgánica es la que proporciona el color obscuro de los suelos, mientras que el resto de los colores son conferidos por las características particulares de la materia mineral (y en particular de la arcilla coloidal). Por lo que las distintas coloraciones observadas en los suelos pueden indicar algunas peculiaridades de sus propiedades: contenido de materia orgánica, grado de permeabilidad y aireación, contenido en determinados minerales, entre otros.

Para el caso particular de la presente investigación, el color de cada una de las muestras fue analizado con una muestra seca al horno y determinado con la notación Munsell. Como se puede observar en la Tabla V, el color tanto del blanco como del tratamiento 1 , es gris muy obscuro, siendo la materia orgánica la principal responsable de este color. El color tanto para el tratamiento 2 como para el tratamiento 3 es negro, siendo la materia orgánica y el carbono de las pilas alcalinas y pilas genéricas las responsables de este color.

El color negro característico para cada una de las muestras en este estudio está en estrecha relación con la materia orgánica en el suelo y con la relación $\mathrm{C} / \mathrm{N}$ alta, según los datos repostados en las respectivas tablas, lo cual indica que la adición de pilas al suelo puede producir las siguientes características en el suelo: falta de oxidación, falta de aireación, lixiviación fuerte de bases, dando una reacción ácida.

TABLA V

COLOR PARA LOS DISTINTOS TRATAMIENTOS SEGÚN LA TABLA MUSELL. COLOR

\begin{tabular}{ccccc}
\hline Lectura & $\mathrm{M}_{1}$ & $\mathrm{M}_{2}$ & $\mathrm{M}_{3}$ & $\mathrm{M}_{4}$ \\
\hline \multirow{4}{*}{1} & Black & Black & Black & Black \\
& $7,5 \mathrm{YR}$ & $10 \mathrm{YR}$ & $5 \mathrm{Y}$ & $10 \mathrm{YR}$ \\
& $2 / 0$ & $2 / 1$ & $2,5 / 2$ & $2 / 1$ \\
\hline \multirow{4}{*}{2} & Very dark gray & Very dark & Very dark & Black \\
& $7,5 \mathrm{YR}$ & gray & gray & $7,5 \mathrm{YR}$ \\
& $3 / 0$ & $10 \mathrm{YR}$ & $5 \mathrm{Y}$ & $2 / 0$ \\
& & $3 / 1$ & $3 / 1$ & \\
\hline \multirow{3}{*}{3} & Dark Brown & Dark gray & Black & Black \\
& $7,5 \mathrm{YR}$ & $10 \mathrm{YR}$ & $10 \mathrm{YR}$ & $5 \mathrm{YR}$ \\
& $3 / 2$ & $4 / 1$ & $2 / 1$ & $2,5 / 1$ \\
\hline
\end{tabular}


Un suelo salino es aquel que presenta una elevada concentración de sales en solución y que muestra las siguientes características:

$\checkmark$ Elevada presión osmótica, por lo que disminuye la disponibilidad de agua para las plantas.

$\checkmark$ Si las sales son de Na además destruyen la estructura del suelo.

Para el caso particular de la presente investigación, el blanco presenta la menor conductividad de todos los tratamientos, con un valor de 0,256 ; le sigue el tratamiento 1 , con una conductividad de 0,3649; luego está el tratamiento 2, con un valor de 0,6997 y finalmente, está el tratamiento 3 con valor de 0,7729 (tabla VI). Esto indica, que la adición de pilas tanto genéricas como alcalinas al suelo hace que la conductividad se incremente como resultado de los procesos de mineralización de los elementos y compuestos químicos aportados por las pilas al suelo, siendo estos datos de conductividad normales, según los datos de referencia del Instituto Geográfico Agustín Codazzi. [13].

TABLA VI

CONDUCTIVIDAD PARA LOS DISTINTOS TRATAMIENTOS A T PROMEDIO DE $25,2^{\circ} \mathrm{C}$.

Conductividad

\begin{tabular}{cccc}
\hline $\mathrm{M}_{1}$ & $\mathrm{M}_{2}$ & $\mathrm{M}_{3}$ & $\mathrm{M}_{4}$ \\
\hline 0,2456 & 0,3649 & 0,6997 & 0,7729 \\
\hline
\end{tabular}

El análisis químico preliminar para cada una de las muestras de suelo objeto de este estudio, se encuentran relacionadas en la Tabla VII. Los resultados se consignaron siguiendo las siguientes convenciones: el signo negativo, indica ausencia del ion o elemento químico y el signo positivo, indica presencia del ion o elemento químico. Según los datos de la Tabla VII, se puede observar que los tratamientos 1,2 y 3 contienen iones cloruros, en cambio en el blanco no se encontraron. Los iones nitratos y sodio se encontraron en todos los tratamientos. Los otros iones analizados: sulfatos, carbonatos, sulfuros, potasio y calcio no fueron identificados en ningún tratamiento.

TABLA VII

IONES EN EXTRACTO ACUOSO PARA LOS DISTINTOS TRATAMIENTOS.

\begin{tabular}{ccccccccc}
\multicolumn{10}{c}{ ONES } \\
\hline $\mathrm{M}$ & $\mathrm{Cl}^{-}$ & $\mathrm{SO}_{4}{ }^{=}$ & $\mathrm{CO}_{3}{ }^{=}$ & $\mathrm{S}^{=}$ & $\mathrm{NO}_{3}{ }^{-}$ & $\mathrm{Na}$ & $\mathrm{K}$ & $\mathrm{Ca}$ \\
\hline $\mathrm{M}_{1}$ & - & - & - & - & + & + & - & - \\
\hline $\mathrm{M}_{2}$ & + & - & - & - & + & + & - & - \\
\hline $\mathrm{M}_{3}$ & + & - & - & - & + & + & - & - \\
\hline $\mathrm{M}_{4}$ & + & - & - & - & + & + & - & - \\
\hline
\end{tabular}

M: tratamientos. $\mathrm{Cl}^{-}$: cloruros; $\mathrm{SO}_{4}{ }^{\prime}$ : sulfatos; $\mathrm{CO}_{3}{ }^{=}$: carbonatos; $\mathrm{S}^{=}$: sulfuros; $\mathrm{NO}_{3}{ }^{-}$: nitratos; Na: sodio; K: potasio; Ca: calcio.

\section{CONCLUSIONES}

El presente estudio permitió hallar elementos importantes en la configuración del suelo suplementado con pilas eléctricas. Aunque no se encontraron cambios sustanciales en el pH sí se evidenció cambios importantes en la relación c/N. De hecho, el carbono orgánico es la principal característica que se modifica en el suelo por adición de pilas, encontrándose mayor incremento en los resultados de las muestras del suelo con pilas alcalinas.

La adición de pilas tanto genéricas como alcalinas al suelo hace que la conductividad se incremente como resultado de los procesos de mineralización de los elementos y compuestos químicos aportados por las pilas al suelo. Por lo anterior, se pudo determinar que el suelo presenta una alta absorción de la energía solar. Esta situación a su vez genera que la temperatura media tienda a ser más elevada, aspecto que provoca una germinación rápida en las semillas en el crecimiento inicial de la planta; no obstante, la presencia de las sustancias contenidas en las pilas eléctricas incide en la baja liberación de nitrógeno, aspecto que afecta directamente la estructura y vitalidad de la planta en sus etapas posteriores a la germinación.

Así mismo, la reducción de las pérdidas de agua por escurrimiento permite deducir que las pilas en el sustrato favorecen de manera importante los procesos de infiltración. De esta manera, se ratifica que las pilas como dispositivos eléctricos afectan en gran medida la capacidad retentiva del agua en el suelo que desencadena degradación del mismo por la generación de procesos de erosión.

\section{AgRADECIMIENTOS}

Agradecimientos especiales a la Universidad de la Amazonia entidad ejecutora del proyecto "Fortalecimiento de las vocaciones científicas en niños, adolescentes y jóvenes mediante la implementación del Programa Ondas en Caquetá", donde mediante convocatoria 2015 cofinanció el proyecto: "Diferencias físicas y químicas que existen en el suelo enriquecido con pilas eléctricas y otro con ausencia de ellas", del cual deriva la presente publicación.

Agradecimiento a los directivos Cesar Augusto Cabrera Muñoz, rector, al profesor Isidoro Rico Murcia, coordinador por su apoyo en la realización del proyecto.

Expresamos nuestro especial agradecimiento a los estudiantes del grado octavo, jóvenes investigadores del grupo: "Pilosos con el Ambiente", de Ondas Colciencias de la Sede San Juan Bosco de la Institución Educativa Antonio Ricaurte y gestores del presente proyecto de investigación.

Al profesor Wilson Rodríguez, coordinador del laboratorio de geociencias del Centro de Investigación Macagual "Cesar Augusto Estrada" de la Universidad de la Amazonia por su apoyo y contribución al proyecto.

A la profesora Claudia González Berrio, Coordinadora de Laboratorios de Química y Biología de la Universidad de la Amazonia por su apoyo y contribución al proyecto.

A la Ingeniera Judy Marcela Dussan, Asesora de Línea del Programa Ondas Colciencias por su apoyo y contribución al proyecto.

\section{REFERENCIAS}

[1] Programa de Naciones Unidas para el Medio Ambiente. Avances y progresos cientificos en nuestro cambiante medio ambiente. Recuperado de http://www.unep.org/yearbook/2010/PDF/UNEP_ES_2010_low.pdf. 2010.

[2] Fernández, A. Basura electrónica, un grave problema ambiental. recuperado de http://www.consumer.es/web/es/medio_ambiente/urbano/2014/03/10/21 9489.php. 2014. 
[3] Díaz, A. Los productores y comercializadores de pilas deberán tener un Sistema de Recolección Selectiva y Gestión Ambiental. Recuperado de http://www.activolegal.com/web/index.php/noticias/actualidad/414noticia180313n1. 2013.

[4] Soto. R. Diagnóstico de la generación de RAEE provenientes del uso de computadores en el sector institucional de Bogotá D.C. (Colombia). Residuos: Revista Técnica. 16 (88), 118-125, 2006.

[5] Martínez, A. Análisis de la generación y disposición de residuos de aparatos eléctricos y electrónicos RAEE'S domiciliarios en el municipio de Yopal (Casanare). Universidad de Manizales. Tesis de maestría. 2015.

[6] Castro, J. and Díaz, M. La contaminación por pilas y baterías en México. Instituto Nacional de Ecología. http://www2.inecc.gob.mx/publicaciones/libros/438/cap5.html. 2007.

[7] Asociación Nacional de Industriales. Investigaciones complementarias. http://www.andi.com.co/pages/proyectos_paginas/contenido.aspx?pro_i $\mathrm{d}=177 \&$ IdConsec $=1427 \&$ clase $=8 \& \mathrm{Id}=20 \& \mathrm{Tipo}=2.2013$.

[8] Robinson, B. E-waste: An assessment of global production and environmental impacts. Science of The Total Environment. 408 (2), 183191. 2009.

[9] Olivetti, E., Gregory, J. and Kirchain, R. Life cycle impacts of alkaline batteries with a focus on end of life. Recuperado de http://www.epbaeurope.net/documents/NEMA_alkalinelca2011.pdf. 2011.

[10] Rydha, C. Life cycle inventory of recycling portable nickel-cadmium batteries. Resources, Conservation and Recycling. 34k(4), 289-309. 2002.

[11] Shen, C., Chen, Y., Huang, S., Wang, Z., Qiao, M., Xu, Y., Setty, K., Zhang, J., Zhu, Y., Lin, Q. Dioxin-like compounds in agricultural soils near e-waste recycling sites from Taizhou area, China: Chemical and bioanalytical characterization. Environment International. 35 (1), 50-55. 2009.

[12] Instituto Geográfico Agustín Codazzi. 1973. Métodos analíticos de laboratorio da suelos. Tercera edición. Bogotá.

[13] Chapman, H. D. Cation Exchange capacity. In: C. A. Black (ed.). Methods of analysis. Part. 2. Agronomy 9. American Society of Agronomy. Madison, Wisconsin. pp. 891-901. 1965.

[14] Jackson M. L. Análisis químico de suelos. Trad. al español por M. J. Beltrán. 3ª edición. Omega. Barcelona, España. 1976.

[15] Allison, L.E. Organic carbon. In: CA Black (ed). Methods of Soil Analysis (Part 2) Chemical and Microbiological Properties. Publisher: America Society of Agronomy Inc., Madison, Wisconsin, USA. Pp: 1367-1378, 1965.

[16] Richards L A. Diagnóstico y rehabilitación de suelos salinos y sódicos. $6^{\text {a }}$. edición. Departamento de Agricultura de Estados Unidos de América. Limusa. México, D.F. 1990.

[17] Moreno, M. J., Belén, D.R., Sánchez, M.P., Viloria, M., García, D. "Evaluación de la actividad antioxidante de extractos de flavonoides de cáscara de naranja en el aceite de soja desodorizado". Interciencia. Vol. 9. no. 29, p.p. 532-538, septiembre de 2004.

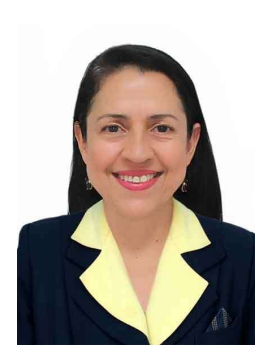

Norma Constanza Bonilla. Nació en Florencia, Caquetá. Licenciada en Química de la Universidad Pedagógica Nacional, Especialista en Educación Básica con Énfasis en Procesos Pedagógicos de la Universidad de la Amazonia y Magíster en Docencia de la Química de la Universidad Pedagógica Nacional. Es docente de la Institución Educativa Jorge Eliecer Gaitán donde enseña Química y Biología en los grados décimo y once. Ha realizado varias investigaciones a nivel escolar con el programa Ondas Colciencias. También es docente catedrática de la Facultad de Ciencias Básicas de la Universidad de la Amazonia, donde enseña Química e investiga en el campo de la actividad biológica de productos naturales a nivel fúngico. Actualmente participa como co-investigadora en el proyecto interinstitucional entre el Instituto Amazónico de Investigaciones Científicas,
SINCHI, la Universidad de la Amazonia y la Asociación de Reforestadores y Cultivadores de Caucho del Caquetá, ASOHECA, en el proyecto: "Ampliación de la base genética del Caucho Natural”.

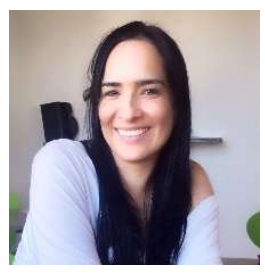

Magda Julissa Rojas Bahamón. Nació en Florencia, Colombia. Se graduó en la Universidad Distrital Francisco José de Caldas como Ingeniera de Sistemas. Realizó una especialización en pedagogía con la Universidad de la Amazonia y posteriormente una maestría en Ciencias de la Educación con la misma universidad. Cursó su Doctorado en Educación y Cultura Ambiental en la Universidad Surcolombiana. Ha desarrollado múltiples investigaciones en el campo de las TIC, los conflictos socioambientales y la educación y la cultura ambiental. Es docente de Tecnología e informática adscrita a la Secretaría de Educación de Florencia. ORCID: 0000-0003-48821476.

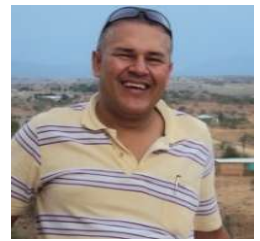

Diego Felipe Arbeláez Campillo. Contador Público de la Universidad de la Amazonia Colombia. Especialista en Revisoría Fiscal Universidad Libre de Colombia. Magister en Ciencias de la Educación Universidad de la Amazonia. Editor de la Revista Amazonia Investiga. ORCID: 0000-0002-9041-9563. 